\title{
PRODUCT INTERACTIONS AND INDUSTRY SHAKEOUT
}

\author{
M. G. O. ESCOBIDO \\ W. Sycip Graduate School of Business, Asian Institute of Management \\ 123 Paseo de Roxas, Makati City, 1260 Philippines \\ mescobido@aim.edu
}

\begin{abstract}
We construct an industry shakeout model based on the Cournot competition of firms each producing a differentiated product and adjusting their output according to the diffusion of their respective product. We investigate the resulting Lotka-Volterra dynamics and present a coexistence region that is a function of the interaction among the products. We show that as the products become less differentiated, the competition intensifies and the coexistence region contracts. The extent of the shakeout depends on the distribution of the competitive interactions among firms in the industry.
\end{abstract}

Keywords: Product differentiation, industry shakeout, Lotka-Volterra dynamics

\section{Introduction}

Over the industry lifecycle, the number of firms rises and then experiences a pronounced decrease or shakeout in the numbers before eventually settling with a few dominant firms. Several models have been advanced to explain the decrease in the number of firms associated with shakeout (see for instance Ref. 1 and references therein). The goal of this paper is to augment the existing literature by presenting a formal model based on the interaction of the products the firms produced.

We consider a Cournot competition of firms each producing a differentiated product, with each firm updating their output based on the diffusion of their respective product. We analyze the co-evolution of the differentiated products and derive the dynamical equations governing their interaction. We show that the resulting dynamical equations are formally equivalent to a Lotka-Volterra competition dynamics.

The paper is organized as follows. The next section presents an industry with two firms each with a competing product. The intent is to highlight the insights that can be gained from the simplest case and provide an illustration of the shakeout dynamics. Then 
we consider $n$-firms and show that the shakeout dynamics persist. We then summarize the results and conclude the paper with opportunities for additional research.

\section{The Model}

We initially consider a duopoly model but this will be extended to competition among $n$ firms in the subsequent section. We follow a market model of Ref. 2 where the (inverse) demand of product $i$ is given by:

$$
p_{i}=p_{i}\left(q_{i}(t), q_{j}(t)\right)=\alpha_{i}-\beta_{i} q_{i}(t)-\gamma_{i j} q_{j}(t) i \neq j=1,2
$$

where $q_{i}$ denote the consumer's consumption of the product $i$ at a price $p_{i}$. The parameter $\alpha_{i}>0$ measures quality in the vertical sense in that an increase in $\alpha_{i}$ increases the marginal utility of consuming product $i$. The symmetric $\gamma_{i j}$ captures the cross-price effect of the differentiation between the products with $\beta_{i}$ denoting the effect from its own quantity. The $\gamma_{i j}$ ranges from $[-1,1]$ depending on whether the products are substitutes $\left(\gamma_{i j}>0\right)$, complements $\left(\gamma_{i j}<0\right)$ or completely differentiated $\left(\gamma_{i j}=0\right)$.

Given the market above, we now consider the competing firms' response. In our case, we use the Cournot model ${ }^{3}$ of competition since the capacity of the firms are assumed difficult to adjust. This means that firms set quantities to maximize their profits knowing the implications on the price. For firm $i$, the revenue in selling $q_{i}$ units of the product at price $p_{i}$ is:

$$
\text { revenue }_{i}\left(q_{i}\right)=p_{i} q_{i}
$$

Assuming a fixed cost $f_{i}$ and a constant marginal cost $m_{i}$, the total cost in the product is:

$$
\operatorname{cost}_{i}\left(q_{i}\right)=f_{i}+m_{i} q_{i} .
$$

Using (2), the profit $\pi_{i}\left(q_{i}\right)$ for the firm $i$ given the output of the other firm $j$ then becomes:

$$
\pi_{i}\left(q_{i}\right)=\left(\theta_{i}-\gamma_{i j} q_{j}\right) q_{i}-f_{i}
$$

where the variable $\theta_{i}=\alpha_{i}-m_{i}$ is the net absolute advantage of the firm $i$ and depends on the quality $(\alpha)$ of its product and the (marginal) cost $(m)$ of producing them.

Maximizing this profit, assuming that the other firms keeps their quantity constant (Cournot conjecture $\partial q_{j} / \partial q_{i}=0$ ), gives the reaction function or best response function of firm $i$ :

$$
q_{i}^{R}\left(q_{j}\right)=\frac{\theta_{i}-\gamma_{i j} q_{j}}{2 \beta_{i}}
$$

The reaction functions intersect to yield a Cournot-Nash equilibrium where each firm will not have an incentive to change its output, given the output of the other firm.

For the firm $i$ to update its initial output $q_{i o}$ to the desired level $q_{i}^{R}\left(q_{j}\right)$ given in (5), it needs to have an adjustment process. Repeating this adjustment process results in a 
dynamic scenario where the firms continuously update their output levels to maximize their profits. The naïve choice of adjustment process is where firms adjust their output level to the discrepancy of the desired output $q_{i}^{R}\left(q_{j}\right)$ and the actual level $q_{i}(t)$ at that instant (i.e. $\left.d q_{i}(t) / d t=q_{i}^{R}\left(q_{j}\right)-q_{i}(t)\right)^{4}$. In this model, the firms ramp to the desired level irrespective of the discrepancy. The resulting dynamics is that the firms adjust toward equilibrium where both coexist. Even a refinement where firms partially adjust their output level in proportion to the discrepancy (i.e. $d q_{i}(t) / d t=q_{i}^{R}\left(q_{j}\right)-$ $q_{i}(t)$ where $r_{i}>0$ ) would still result in the coexistence of firms ${ }^{5}$.

Instead of the partial adjustment process, we posit that firms update their output following the diffusion of their product. Since the firm $i$ is not going to produce more than $q_{i}^{R}\left(q_{j}\right)$ units, the diffusion can be modeled by a logistic function with $q_{i}^{R}\left(q_{j}\right)$ as the asymptotic limit. This yields an adjustment process given by a logistic equation:

$$
\frac{d q_{i}(t)}{d t}=r_{i}\left(q_{i}^{R}\left(q_{j}\right)-q_{i}(t)\right) q_{i}(t) .
$$

The $r_{i}$ controls the speed of diffusion of the product - the larger it is, the faster the market is in adopting the product and the steeper the firm has to ramp its output. Compared to the partial adjustment process, the rate of change of the output is not constant but changes as the output change.

If the firms adopt the logistic adjustment process, from (5) and (6), the resulting dynamical equations become:

$$
\frac{d q_{i}(t)}{d t}=r_{i} q_{i}(t)\left(\frac{\alpha_{i}-m_{i}}{2 \beta_{i}}-q_{i}(t)-\frac{\gamma_{i j}}{2 \beta_{i}} q_{j}(t)\right) .
$$

If we let

$$
\begin{gathered}
M_{i}=\frac{\theta_{i}}{2 \beta_{i}}=\frac{\alpha_{i}-m_{i}}{2 \beta_{i}} \\
c_{i j}=\frac{\gamma_{i j}}{2 \beta_{i}} .
\end{gathered}
$$

Then

$$
\frac{d q_{i}(t)}{d t}=r_{i} q_{i}(t)\left(M_{i}-q_{i}(t)-c_{i j} q_{j}(t)\right)
$$

Equation (9) is formally equivalent to Lotka-Volterra competition equations popular in population ecology (see for instance Refs. 6-7). The derivation, however, departs from the usual tack where the equations are used directly and a conjecture is offered as to what the variables might represent in the new context $t^{8-9}$. In our case, the rate of growth $r_{i}$ would pertain to the growth of product $i$ 's market; the carrying capacity would just be the monopoly output $M_{i}$ and would depend on the firm advantage $\theta_{i}$; and the competition coefficient $c_{i j}$ emerges as a consequence of the differentiation among the products and the self-price effect. 


\section{Competition Dynamics}

The dynamics of the coupled equations (9) can be understood by examining the terms to the right. The third term involves $c_{i j} q_{i} q_{j}$ and denotes the interaction between the products. If the products are perfectly differentiated $\left(\gamma_{i j}=0\right)$, then $c_{i j}=0$ and the contribution of this term is zero. This means the products do not interact and the output initially grows exponentially at the rate $r_{i} M_{i}$. The largest its output becomes would be its monopoly output $M_{i}$. As the output grows, however, the second term $q_{i}^{2}$ becomes larger and decreases the growth rate. This happens because the discrepancy to the monopoly output $M_{i}$ becomes less as there are fewer customers left to target.

The above dynamics will result in the output tracing an S-curve over time: gradually increasing as more units become available and then slowing down. The maximum desired level it is going to produce is its monopoly output $M_{i}=\theta_{i} / 2 \beta_{i}$. Both the rate of growth and the monopoly output is directly proportional to the firm's net absolute advantage. As such the firm can increase how fast it is growing and how many it is going to produce by improving its net absolute advantage.

If the products are substitutes $\left(\gamma_{i j}>0\right)$, then the competition coefficient is positive $\left(c_{i j}>0\right)$. The effect of the competition by other products would be to reduce the growth rate of the production of $q_{i}$ 's. As such, as the products become less differentiated (higher $\gamma_{i j}$ ), the impact of competition becomes more intense (higher $c_{i j}$ ) and reduces the growth rate of the production of the affected products. The continued reduction in growth rate past certain thresholds may eventually lead some firms to exit the market. Whether this would be sufficient for an industry shakeout would depend on the distribution of competitive interactions among the firms in the industry.

Given the competition setting, it would be insightful to determine the output which would not vary over time. These equilibrium outputs $\left(q_{i}^{*} \mathrm{~s}\right)$ can be obtained by setting $d q_{i}(t) / d t=0$ and the resulting combination $\left(q_{i}^{*}, q_{j}^{*}\right)$ would become points in the phase space covering $q_{i}$ and $q_{j}$. For these equilibrium points to persist, we need to check their stability. For a duopoly, the condition for the stability of the coexistence of the products can be obtained by considering (9) with $q_{1} \approx 0$. When $q_{1}$ is small, $q_{2}$ approaches its equilibrium level $q_{2}^{*}$ which is just its monopoly output $M_{2} \cdot q_{1}$ will grow provided $M_{1}>c_{12} q_{2}^{*}$. Similarly, $q_{2}$ when small, will grow provided $M_{2}>c_{21} M_{1}$. As such, both firms can coexist if $c_{12}<M_{1} / M_{2}$ and $c_{21}<M_{2} / M_{1}$.

The evolution of the competition would vary depending on which condition the parameters of the system satisfy. Although there are infinite possible paths in the actual evolution depending on the parameters and initial conditions, there are, however, only four long-run outcomes - the trivial equilibrium point $(0,0)$, one firm dominates the market and the other exits $\left(\left(M_{1}, 0\right)\right.$ or $\left.\left(0, M_{2}\right)\right)$ or both firms coexist $\left(\left(M_{1}-c_{12} M_{2}\right) /(1-\right.$ $\left.\left.c_{12} c_{21}\right),\left(M_{2}-c_{21} M_{1}\right) /\left(1-c_{12} c_{21}\right)\right)$. The results are summarized in Table 1.

In terms of the firms' net absolute advantage, the conditions for stable coexistence can be cast as $\theta_{1}>\left(\gamma_{12} \theta_{2}\right) /\left(2 \beta_{2}\right)$ and $\theta_{2}>\left(\gamma_{21} \theta_{1}\right) /\left(2 \beta_{1}\right)$. If $\beta_{1}=\beta_{2}=\beta$, and $\gamma_{12}=\gamma_{21}=\gamma$ then $\gamma /\left(2 \beta_{i}\right)<1$, and $\theta_{i}>\left(\gamma \theta_{j}\right) /\left(2 \beta_{j}\right), i \neq j$ will lie to the left $\left(\theta_{1}\right)$ or right $\left(\theta_{2}\right)$ of the $\theta_{2}=\theta_{1}$ diagonal. 
Table 1. Equilibrium points and their stability

\begin{tabular}{|c|c|c|}
\hline $\begin{array}{l}\text { Equilibrium Points } \\
\qquad\left(\boldsymbol{q}_{\mathbf{1}}^{*}, \boldsymbol{q}_{\mathbf{2}}^{*}\right)\end{array}$ & Eigenvalues & Stable if \\
\hline$(0,0)$ & $\begin{array}{l}\lambda_{1}=r_{1} M_{1} \\
\lambda_{1}=r_{2} M_{2}\end{array}$ & Never \\
\hline$\left(M_{1}, 0\right)$ & $\begin{array}{l}\lambda_{1}=-r_{1} M_{1} \\
\lambda_{2}=-r_{2}\left(c_{21} M_{1}-M_{2}\right)\end{array}$ & $c_{21}>\frac{M_{2}}{M_{1}}$ or $\theta_{2}<\frac{\gamma_{12}}{2 \beta_{1}} \theta_{1}$ \\
\hline$\left(0, M_{2}\right)$ & $\begin{array}{l}\lambda_{1}=-r_{2} M_{2} \\
\lambda_{2}=-r_{1}\left(c_{12} M_{2}-M_{1}\right)\end{array}$ & $c_{12}>\frac{M_{1}}{M_{2}}$ or $\theta_{1}<\frac{\gamma_{12}}{2 \beta_{2}} \theta_{2}$ \\
\hline$\left(\begin{array}{c}\frac{M_{1}-c_{12} M_{2}}{1-c_{12} c_{21}} \\
\frac{M_{2}-c_{21} M_{1}}{1-c_{12} c_{21}}\end{array}\right)$ & $\begin{array}{l}\lambda_{ \pm}=-\frac{a_{1}+a_{2}}{2} \pm \\
\frac{\sqrt{\left(a_{1}+a_{2}\right)^{2}-4\left(1-c_{12} c_{21}\right) a_{1} a_{2}}}{2} \\
a_{i}=r_{i} q_{i}^{*}\end{array}$ & $c_{12}<\frac{M_{1}}{M_{2}}$ and $c_{21}<\frac{M_{2}}{M_{1}}$ \\
\hline
\end{tabular}

There are then three competition zones corresponding to the different conditions with which a firm gains a monopoly or when the firms coexist. The figure below illustrates this:

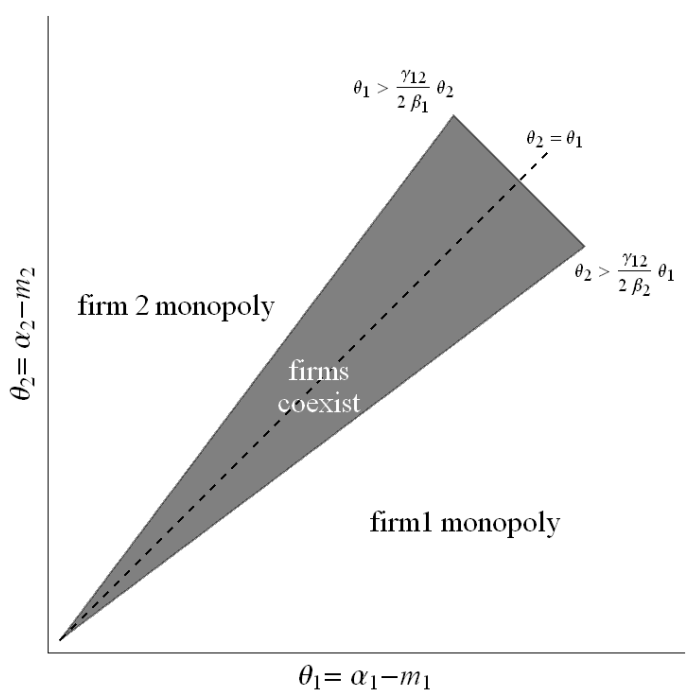

Fig. 1. Firms' net absolute advantage and competition zones

The visualization of the conditions above illustrates the dynamics as the products evolve from being completely differentiated to completely substitutable. Usually, early in the industry life cycle, market needs are addressed through highly differentiated products. The middle region in Fig. 1 would show a coexistence region that is large (low $\gamma$ ). Over time, however, the differentiation among products can decrease, intensifying the competition and contracting further the region of coexistence. The extreme scenario 
would be when the products are completely substitutable $(\gamma=1)$, wherein the coexistence region is the smallest and possibly will collapse.

To illustrate the interaction dynamics when products become completely undifferentiated, consider the case where the firms have the same parameters (i.e. $\alpha_{1}=\alpha_{2}=\alpha, m_{1}=m_{2}=m$ and $\beta_{1}=\beta_{2}=0.5$ ) resulting in a symmetric competition with $c_{i j}=\gamma$. The equilibrium points and their condition of stability are simplified as shown in Table 2.

Table 2. Equilibrium points and their stability for the case $M_{1}=M_{2}=M ; \beta_{1}=\beta_{2}=0.5$

\begin{tabular}{|l|c|}
\hline $\begin{array}{c}\text { Equilibrium Points } \\
\left(\boldsymbol{q}_{\mathbf{1}}^{*}, \boldsymbol{q}_{2}^{*}\right)\end{array}$ & Stable if \\
\hline$(0,0)$ & Never \\
\hline$(M, 0)$ & $c=\gamma>1$ \\
\hline$(0, M)$ & $c=\gamma>1$ \\
\hline$\left(\frac{M}{1+c}, \frac{M}{1+c}\right)$ & $c=\gamma<1$ \\
\hline
\end{tabular}

Plotting the relations in Table 2 as the competition increases shows a transition from coexistence of the firms to one where the other is being shaken out of the market. This result is shown in Fig. 2. The shakeout results in one firm dominating and driving the other to exit the market. In the next section, we will consider the case where there are more firms and see if such dynamics persist.

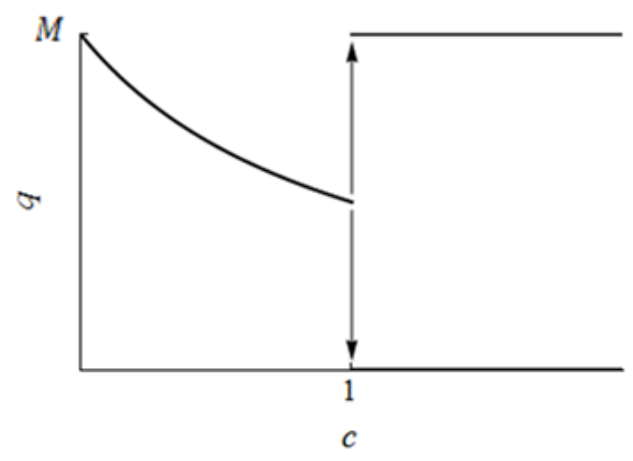

Fig. 2. Transition from coexistence to where one is shaken-out as competition increases

\section{4. n-Firms}

To model industry shakeout, we now consider the generalization to $n$-firms each producing a differentiated product. The generalization of (9) to $n$ competing firms becomes: 


$$
\frac{d q_{i}(t)}{d t}=r_{i} q_{i}\left(M_{i}-q_{i}-\sum_{j \neq i}^{n} c_{i j} q_{j}\right)
$$

For $n$ firms, there are $2^{n}$ equilibrium points for which $\dot{q}_{l}(t)$ is zero - either $q_{i}=0$ or $M_{i}-q_{i}-\sum_{j \neq i}^{n} c_{i j} q_{j}=0$. Following Ref. 10, we can investigate the equilibrium solutions $\left(q_{1}^{*}, q_{2}^{*}, \ldots, q_{k}^{*}, 0, \ldots, 0\right)$ with the first $q_{i}^{*}$ 's non-zero and the remaining $n-k$ zero. We then consider the cases where $k=0,1,1<k<n$ and $k=n$.

For simplicity, we assume the same parameters for all firms (i.e. $M_{i}=M_{j}$ ) and the interaction symmetric and constant (i.e. $c_{i j}=c_{j i}=c$ ). The value then of $q_{i}^{*}$ for $1 \leq i \leq$ $k$ will be given by the solution of :

$$
\left[\begin{array}{ccccc}
1 & c & c & \ldots & c \\
c & 1 & c & \ldots & c \\
c & c & 1 & \ldots & c \\
\vdots & \vdots & \vdots & \ddots & \vdots \\
c & c & c & \ldots & 1
\end{array}\right]\left[\begin{array}{c}
q_{1}^{*} \\
q_{2}^{*} \\
q_{3}^{*} \\
\vdots \\
q_{k}^{*}
\end{array}\right]=\left[\begin{array}{c}
M \\
M \\
M \\
\vdots \\
M
\end{array}\right] .
$$

The matrix can be inverted to give $q_{i}^{*}=M /(1+(k-1) c)$. As such,

$$
q_{i}^{*}=\left\{\begin{array}{cc}
\frac{M}{1+(k-1) c} & 1 \leq i \leq k \\
0 & k+1 \leq i \leq n .
\end{array}\right.
$$

Given the values above, the Jacobian about this equilibrium takes the form:

$$
\boldsymbol{J}=\left[\begin{array}{ccccccc}
X & Y & \ldots & Y & 0 & \ldots & 0 \\
Y & X & \ldots & Y & 0 & \ldots & 0 \\
\vdots & \vdots & \ddots & \vdots & \vdots & \ddots & \vdots \\
Y & Y & \ldots & X & 0 & \ldots & 0 \\
0 & 0 & \ldots & 0 & Z & \ldots & 0 \\
\vdots & \vdots & \ddots & \vdots & \vdots & \ddots & \vdots \\
0 & 0 & \ldots & 0 & 0 & \ldots & Z
\end{array}\right]
$$

where

$$
\begin{aligned}
& \left.\frac{\partial \dot{q}_{l}}{\partial q_{i}}\right|_{\left(q_{i}^{*}, q_{j}^{*}\right)}=\left\{\begin{array}{cc}
X=-\frac{r M}{1+(k-1) c} & 1 \leq i \leq k \\
Z=\frac{r M(1-c)}{1+(k-1) c} & k+1 \leq i \leq n
\end{array}\right. \\
& \left.\frac{\partial \dot{q}_{l}}{\partial q_{j}}\right|_{\left(q_{i}^{*}, q_{j}^{*}\right)}=\left\{\begin{array}{cc}
Y=-\frac{r c M}{1+(k-1) c} & 1 \leq i \leq k \\
0 & k+1 \leq i \leq n .
\end{array}\right.
\end{aligned}
$$

The characteristic equation can be readily solved by noting that (13) is a block matrix. As such 


$$
|\boldsymbol{J}-\lambda \boldsymbol{I}|=(Z-\lambda)^{n-k}(\lambda-(X-Y))^{k-1}(\lambda-(X+(k-1) Y)) .
$$

The eigenvalues can then be extracted as:

$$
\begin{gathered}
\lambda_{1}=Z=\frac{r M(1-c)}{1+(k-1) c} \text { with multiplicity }(n-k) \\
\lambda_{2}=X-Y=-\frac{r M(1-c)}{1+(k-1) c} \text { with multiplicity }(k-1) \\
\lambda_{3}=X+(k-1) Y=-r M
\end{gathered}
$$

The other cases can be read of from the derivation above. The results are summarized in the table below:

Table 3. $\boldsymbol{n}$-firms equilibrium points and stability

\begin{tabular}{|l|l|l|c|}
\hline \multicolumn{1}{|c|}{$k$} & $\begin{array}{c}\text { Equilibrium Points } \\
\left(q_{1}^{*}, q_{2}^{*}, \ldots, q_{k}^{*}, \ldots, q_{n}^{*}\right)\end{array}$ & \multicolumn{1}{|c|}{ Eigenvalues } & Stable if \\
\hline 0 & $(0,0, \ldots, 0)$ & $\lambda_{1}=r M$ & Never \\
\hline 1 & $\left(q_{1}^{*}, 0, \ldots, 0\right)$ & $\begin{array}{l}\lambda_{1}=-r M \\
\lambda_{2}=r(1-c) M\end{array}$ & $c>1$ \\
\hline $1<k<n$ & $\left(q_{1}^{*}, q_{2}^{*}, \ldots, q_{k}^{*}, 0, \ldots, 0\right)$ & $\lambda_{1}=\frac{r M(1-c)}{1+(k-1) c}$ & Never \\
& & $\begin{array}{l}\lambda_{2}=-\frac{r M(1-c)}{1+(k-1) c} \\
\lambda_{3}=-r M\end{array}$ & $c<1$ \\
\hline$n$ & $\left(q_{1}^{*}, q_{2}^{*}, \ldots, q_{n}^{*}\right)$ & $\lambda_{1}=-\frac{r M(1-c)}{1+(n-1) c}$ & \\
\hline
\end{tabular}

The results summarized in Table 3 show that $n$-firms coexist as long as the competition coefficient is less than one. As the products become more substitutable and less differentiated, the competition coefficient approaches one and eventually shaking the other firms out of the industry. As such, the shakeout dynamics observe in the duopoly case persists even if there are more firms involved, at least in our simplified, symmetric configuration.

Analytically solving the coupled differential equations beyond the symmetric configuration is not in general possible. For illustration, we are going to simulate the resulting dynamics for different distribution of product interactions and show that the extent of industry shakeout depends on this.

We consider a truncated normal distribution of $n(n-1) / 2$ independent product interactions in the range $[0,1]$ for the $n$-firms in the industry. If the products are largely 
differentiated, the mean of the differentiation coefficient would be close to zero (see Fig. 3.a below). As the differentiation becomes less and the substitutability increases, the mean of the distribution increases toward one.

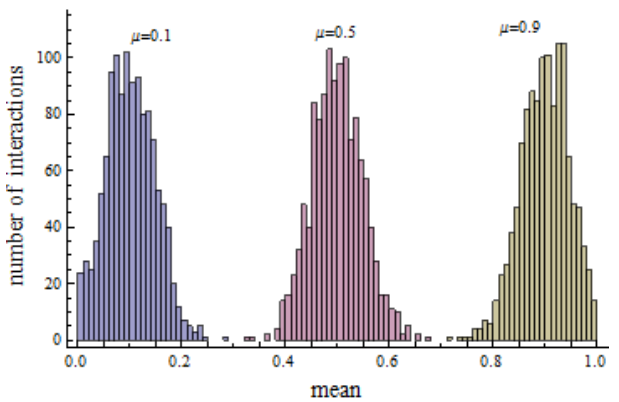

a.) Distributions of the differentiation coefficients

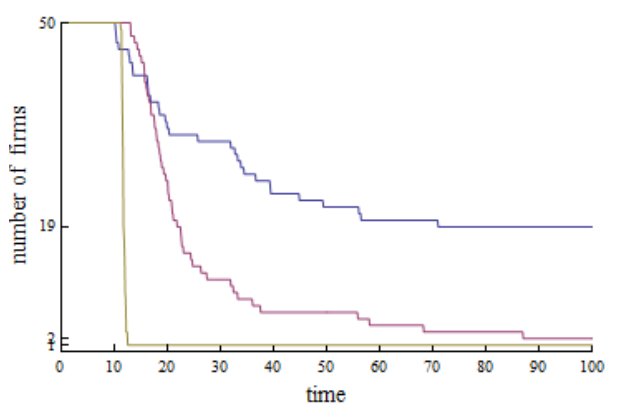

b.) Evolution in the number of firms over time

Fig. 3. Industry shakeout as a function of the distribution of product differentiation $(\sigma=0.1)$

The resulting evolution of an industry with 50-firms is depicted in Fig. 3.b. As the mean $\mu$ of the distribution of the differentiation coefficients increases from 0.1 to 0.9 , the extent of the shakeout becomes more pronounced. The transition from all the firms coexisting to firms being shaken-out of the industry or until one firm survives becomes steeper.

\section{Conclusion}

By considering the Cournot competition of firms each with a differentiated product and adjusting their output based on the diffusion of their respective product, we were able to show that the resulting Lotka-Volterra dynamics can result in an industry shakeout. For the duopoly case, we visualize the dynamics by presenting a coexistence region on which firms coexist depending on the interaction of their products along with their respective relative net absolute advantages. As the products become less differentiated, the competition becomes more intense and the coexistence region contracts. Simulation of different distributions of the product interaction showed that the extent of the shakeout becomes more pronounced as the products becomes less and less differentiated.

More insights can be extracted from the model by considering other cases. For instance, the effect of market growth can be investigated by simulating different rates of diffusion and the net absolute advantage of firms can be varied and made to evolve over time. Time dependence, the stochastic nature and delays prevalent in the interactions can add more realism to the model. This is being covered in other articles. The insight, however, on how product interactions affect industry dynamics adds to the understanding of industry shakeouts. 


\section{Acknowledgement}

The author wants to thank Prof. Christopher Bernido and Prof. M. Victoria CarpioBernido for the support and the opportunity to participate in the conference. Assistance from the Asian Institute of Management is gratefully acknowledged.

\section{References}

1. S. Klepper and K.L. Simons, Industry shakeouts and technological change, International Journal of Industrial Organization 23:23-43 (2005).

2. A. Dixit, A Model of Duopoly Suggesting a Theory of Entry Barriers, Bell Journal of Economics 10:20-32 (1979).

3. A. Cournot,. Recherches sur les Principes Mathematiques de la Theorie des Richesses, Paris (1838).

4. R.D. Theocharis, On the Stability of the Cournot Solution on the Oligopoly Problem, The Review of Economic Studies 27(2):133-134 (1960).

5. F. Fisher, The Stability of the Cournot Oligopoly solution: The Effects of Speeds of Adjustment and Increasing Marginal Costs, The Review of Economic Studies 28(2):125-135 (1961).

6. N.S. Goel, S.C. Maitra, and E.W. Montroll, On the Volterra and Other Nonlinear Models of Interacting Populations, Reviews of Modern Physics 43(2):231-276 (1971).

7. J.D. Murray, Mathematical Biology, Berlin: Springer (1989).

8. O. Malcai, O. Biham, P. Richmond, S. Solomon, Theoretical analysis and simulations of the generalized Lotka-Volterra model, Physical Review E 66:031102 (2002).

9. Bhargava S.C., Generalized Lotka-Volterra Equations and the Mechanism for Technological Substitution, Technological Forecasting and Social Change 35:319-326 (1989).

10. S.M. Sebastian and B.A. Huberman, Competitive Dynamics of Web Sites, Journal of Economic Dynamics and Control 27(11):2195-2206 (2003). 